\title{
The impact of the gate dielectric quality in developing Au-free D-mode and E-mode recessed gate AlGaN/GaN transistors on a $200 \mathrm{~mm}$ Si substrate
}

\author{
Tian-Li Wu ${ }^{1,2}$, Denis Marcon ${ }^{1}$, Brice De Jaeger ${ }^{1}$, Marleen Van Hove ${ }^{1}$, Benoit Bakeroot ${ }^{1,3}$, Dennis Lin $^{1}$, Steve \\ Stoffels ${ }^{1}$, Xuanwu Kang ${ }^{1}$, Robin Roelofs ${ }^{4}$, Guido Groeseneken ${ }^{1,2}$, and Stefaan Decoutere ${ }^{1}$ \\ ${ }^{1}$ imec, Kapeldreef 75, Leuven, Belgium \\ ${ }^{2}$ Department of Electrical Engineering, KU Leuven, Belgium \\ ${ }^{3}$ Center for Microsystem Technology, Ghent University, Belgium \\ ${ }^{4}$ ASM, Kapeldreef 75, Leuven, Belgium \\ Phone:+32 (0) 16-283492, Tian-Li.Wu@imec.be
}

\begin{abstract}
The selection of the gate dielectric is one of the most critical stability issues in recessed gate $\mathrm{AIGaN} / \mathrm{GaN}$ transistors. In this work, we show that the quality of the gate dielectric has a strong impact on: 1$)$ the threshold voltage $\left(\mathbf{V}_{\mathrm{TH}}\right)$ hysteresis, 2) the drain current reduction for enhancement mode devices, and 3) the forward gate bias TDDB (time dependent dielectric breakdown). It will be shown that the $V_{T H}$ hysteresis and the current reduction can be minimized by using a dielectric with lower interface state density $\left(\mathrm{D}_{\mathrm{it}}\right)$ and less border traps, e.g., a PE-ALD SiN dielectric. Furthermore, the $0.01 \%$ failures at 20 years TDDB requirement at $150^{\circ} \mathrm{C}$ for a large power device, e.g., gate width $W g=36 \mathrm{~mm}$, necessitates the use of at least a $25 \mathrm{~nm}$ thick PE-ALD SiN gate dielectric.
\end{abstract}

Keywords-AlGaN/GaN; recessed gate; gate dielectric; PEALD SiN; interface states; border traps; depletion mode; enhacement mode

\section{INTRODUCTION}

AlGaN/GaN Metal Insulator Semiconductor High Electron Mobility Transistors (MIS-HEMTs) are considered as promising candidates for power switching applications due to a low gate leakage current [1]. A recessed gate process is often used to achieve an E-mode characteristic [2][3][4][5]. Then, the properties of the interface and the quality of the gate dielectric become the most challenging reliability issues. Recently, high quality gate dielectrics in D-mode [6][7][8] and E-mode [3][4][5][9] devices have been demonstrated and characterized. However, the detailed analysis of the correlation of interface states/border traps and device characteristics is still missing. In this work, the impact of the interface properties and the quality of the gate dielectrics on both D-mode and E-mode device characteristics is extensively evaluated in recessed gate transistors with three different recess depths. A frequency dependent conductance analysis and an $\mathrm{AC}$ transconductance $\left(\mathrm{AC}-\mathrm{g}_{\mathrm{m}}\right)$ technique are used to examine the interface states and the border traps for different gate dielectrics. These analyses are then correlated to the electrical characteristics, i.e., the threshold voltage $\left(\mathrm{V}_{\mathrm{TH}}\right)$ hysteresis and the drain current. The physical mechanisms to explain these results are proposed. Finally, the time dependent dielectric breakdown (TDDB) methodology is used to evaluate the strength of the gate dielectric and predict the lifetime.

\section{DEVICE FABRICATION}

All samples were fabricated with a Au-free CMOS compatible process on $200 \mathrm{~mm} \mathrm{Si}$ (111) wafers, starting with an AlN nucleation layer, a $2.3 \mu \mathrm{m}$ AlGaN buffer, a $150 \mathrm{~nm}$ $\mathrm{GaN}$ channel, a $15 \mathrm{~nm} \mathrm{Al}_{0.25} \mathrm{Ga}_{0.75} \mathrm{~N}$ barrier, and a $3 \mathrm{~nm} \mathrm{GaN}$ cap layer. Three different recess depths of AlGaN barrier are listed in Table1. A recessed gate is commonly used to reduce the 2DEG under the gate, leading to an enhancement mode characteristic. The schematics of the devices with three different recessed gate depth are shown in Fig. 1 and Fig. 7. The summary of the important process parameters is shown in Table. 1. Two different gate dielectrics, Plasma-Enhanced ALD (PE-ALD) SiN deposited in an ASM system, and Rapid Thermal CVD (RTCVD) SiN were used as a gate dielectric on these devices after a HF2\% clean.

Table 1. Summary of important process parameters

\begin{tabular}{l|c|c}
\hline \multicolumn{1}{c|}{ Device } & $\begin{array}{c}\text { Recess depth } \\
\text { (AlGaN barrier) }\end{array}$ & $\begin{array}{c}\text { Gate dielectric } \\
\text { thickness }\end{array}$ \\
\hline D-mode MIS-HEMT & $14.3 \mathrm{~nm}(3.7 \mathrm{~nm})$ & $15 \mathrm{~nm}$ \\
E-mode MIS-HEMT & $16.5 \mathrm{~nm}(1.5 \mathrm{~nm})$ & $25 \mathrm{~nm}$ \\
E-mode MIS-FET & $21.7 \mathrm{~nm}(-3.7 \mathrm{~nm} *)$ & $25 \mathrm{~nm}$ \\
\hline
\end{tabular}

*-3.7nm remaining $\mathrm{AlGaN}$ barrier indicates $3.7 \mathrm{~nm}$ etching depth into the GaN channel.

\section{RESULTS AND DISCUSSIONS}

\section{A. Recessed gate D-mode AlGaN/GaN transistor:}

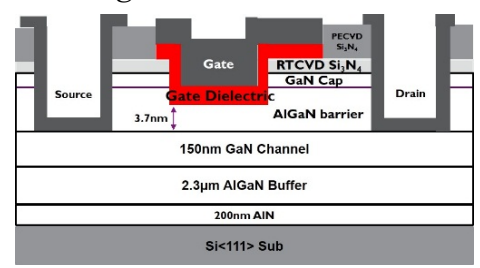

Fig. 1. Schematic cross-section of a D-mode recessed gate MIS-HEMT with $3.7 \mathrm{~nm}$ remaining AlGaN barrier. 
In order to characterize the interface properties of the gate region between the gate dielectric and the $\mathrm{AlGaN}$ barrier shown in Fig. 1, a frequency dependent conductance analysis was done on a capacitor to extract the interface state density $\left(D_{i t}\right)$ [10]. A positive gate bias is needed to transfer the electrons from the channel to the interface between the AlGaN barrier and the gate dielectric to interact with the interface states. Therefore, the following conditions are measured on the capacitor structures: $\mathrm{V}_{\mathrm{G}}=2.4 \mathrm{~V} \sim 3.4 \mathrm{~V}$ (RTCVD SiN) and $\mathrm{V}_{\mathrm{G}}=3 \mathrm{~V} \sim 4 \mathrm{~V}$ (PE-ALD SiN). In this case, please note that we consider the electrons only interact with the interface state rather than the border traps, which will be discussed in the next paragraph. Fig. 2 shows the extracted $D_{i t}$ values at the interface between the gate dielectric and the AlGaN barrier. The $\mathrm{D}_{\text {it }}$ is significantly lower for the device with a PE-ALD SiN $\left(D_{\text {it }} \sim 1 \times 10^{11}-2.3 \times 10^{12} \mathrm{~cm}^{-2} \mathrm{eV}^{-1}\right)$ gate dielectric than for the device with an RTCVD SiN ( $D_{\mathrm{it}} \sim 3.3 \times 10^{13}-3.5 \times 10^{13} \mathrm{~cm}^{-}$ ${ }^{2} \mathrm{eV}^{-1}$ ) gate dielectric.

Furthermore, these devices were further characterized using the $\mathrm{AC}$ transconductance (AC-g $\mathrm{g}_{\mathrm{m}}$ ) technique (Fig. 3). This technique examines the effect of border traps on the carrier transport and the amount of $\mathrm{AC}-\mathrm{g}_{\mathrm{m}}$ dispersion reflects the border trap density in the gate dielectric [11]. A significantly lower $g_{m}$ peak dispersion was observed in the device with a PE-ALD SiN gate dielectric compared with the device with an RTCVD SiN (Fig. 4) gate dielectric. Fig. 5 shows the typical $I_{D}-V_{G}$ characteristics with a double $V_{G}$ sweep. Under a positive gate voltage, the conduction band of the $\mathrm{AlGaN}$ barrier is pulled down.

Based on the aforementioned characterizations, the electrons can be transferred from the channel to the interface between the gate dielectric and the AlGaN barrier, where they are trapped by interface states or border traps (Fig. 6), leading to the $\mathrm{V}_{\mathrm{TH}}$ hysteresis. Again, the device with a PE-ALD SiN gate dielectric shows less hysteresis compared with the other one. According to [12], the plasma enhanced deposition results in a better surface reaction due to additional energy from the ion bombardment in the plasma step. Therefore, in order to minimize the hysteresis, a good dielectric quality with a lower $\mathrm{D}_{\mathrm{it}}$ and less border traps is mandatory.

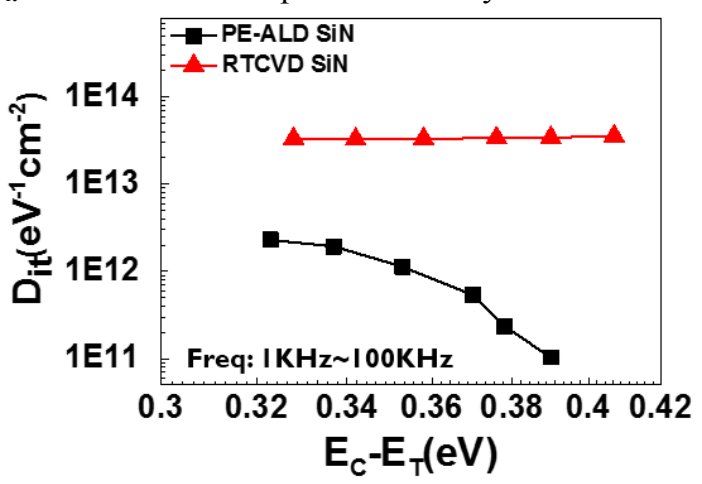

Fig. 2. $D_{i t}$ measurement of MIS-HEMTs with $3.7 \mathrm{~nm}$ remaining AlGaN barrier on a capacitor. The trap state energy was estimated based on the Shockley-Read-Hall statistical model with a capture cross-section $1 \times 10^{-15} \mathrm{~cm}^{2}$.

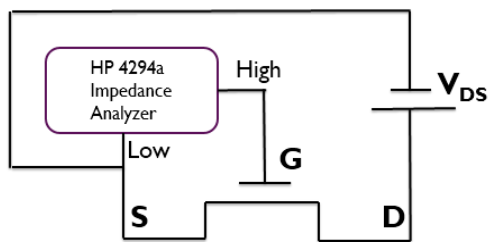

Fig. 3. Diagram of the AC- $g_{m}$ measurement setup. A dc bias mixed with an ac sinusoidal signal $\left(\mathrm{V}_{\mathrm{rms}}=20 \mathrm{mV}\right)$ is applied on the gate while a small dc bias $(50 \mathrm{mV})$ is applied on the drain to inject the carriers across the channel.

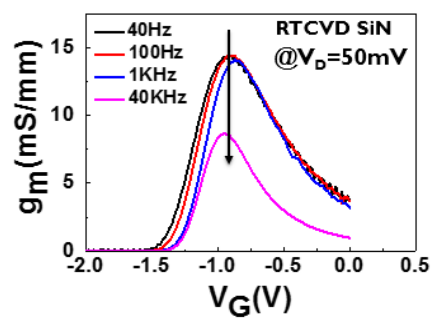

(a)

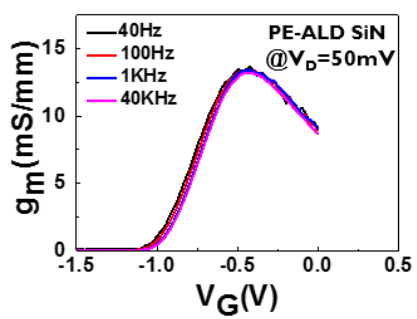

(b)
Fig. 4. $g_{m}$ dispersion characteristics for $3.7 \mathrm{~nm}$ remaining AlGaN barrier with an RTCVD SiN (a), or a PE-ALD SiN (b) gate dielectric.

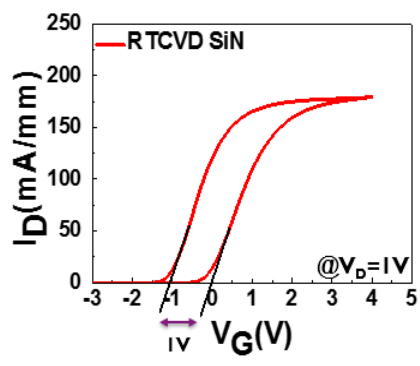

(a)

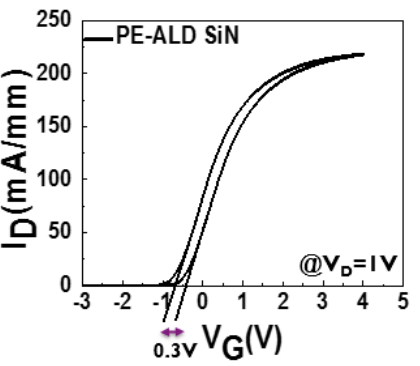

(b)
Fig. 5. $\mathrm{I}_{\mathrm{D}}-\mathrm{V}_{\mathrm{G}}$ characteristics of the devices with $3.7 \mathrm{~nm}$ remaining AlGaN barrier with an $\mathrm{RTCVD} \operatorname{SiN}\left(\mathrm{V}_{\mathrm{TH}}=-1.3 \mathrm{~V}\right)$ (a), or a PE-ALD SiN $\left(\mathrm{V}_{\mathrm{TH}}=-1 \mathrm{~V}\right)(\mathrm{b})$ gate dielectric. The $\mathrm{V}_{\mathrm{TH}}$ hysteresis is $1 \mathrm{~V}$ and $0.3 \mathrm{~V}$, respectively.

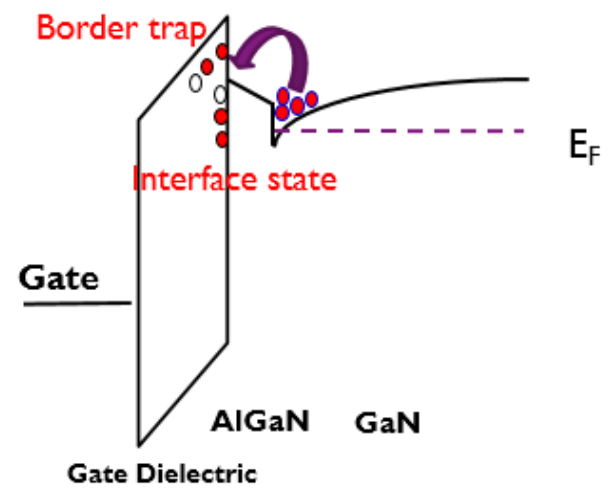

Fig. 6. Schematic band diagram indicating that electrons can be trapped by the interface states or the border traps under a positive gate bias condition. 
B. Recessed gate E-mode AlGaN/GaN transistors:

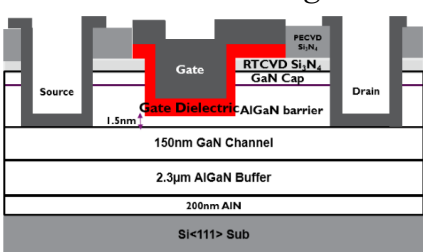

(a)

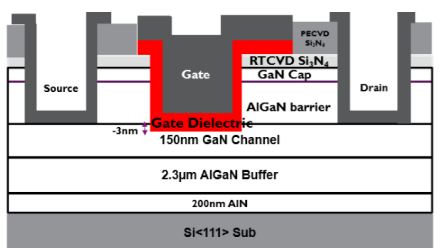

(b)
Fig. 7. Schematic cross-section of the E-mode recessed gate MIS-FETs with $1.5 \mathrm{~nm}$ remaining $\mathrm{AlGaN}$ barrier (a) and $3.7 \mathrm{~nm}$ etch depth into the GaN channel (b).

Fig. 7 shows the schematics of the E-mode transistors with two different remaining $\mathrm{AlGaN}$ barrier thickness under the gate region. Fig. 8 shows the $\mathrm{I}_{\mathrm{D}}-\mathrm{V}_{\mathrm{G}}$ characteristics and the transconductance $\left(g_{m}\right)$ for devices with a $1.5 \mathrm{~nm}$ remaining AlGaN barrier. Considering a D-mode and an E-mode device, the device with an RTCVD SiN gate dielectric shows a more pronounced current drop and $\mathrm{g}_{\mathrm{m}}$ degradation compared to the one with a PE-ALD SiN gate dielectric
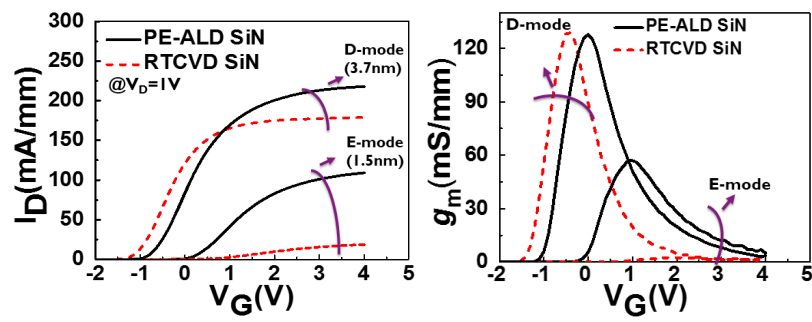

Fig. 8. $I_{D}-V_{G}$ and $g_{m}-V_{G}$ characteristics of the devices with $1 \mathrm{~nm}$ remaining $\mathrm{AlGaN}$ barrier. The $\mathrm{V}_{\mathrm{TH}}$ is $0 \mathrm{~V}$ and $0.6 \mathrm{~V}$, respectively for a PE-ALD $\mathrm{SiN}$ and an RTCVD $\mathrm{SiN}$ gate dielectric.

To increase the $\mathrm{V}_{\mathrm{TH}}$, the AlGaN barrier can be completely recessed into the GaN channel, resulting in an E-mode MISFET rather than a MIS-HEMT (Fig. 7(b)). Fig. 9 shows the $I_{D}-$ $\mathrm{V}_{\mathrm{G}}$ and the $\mathrm{g}_{\mathrm{m}}$ of the recessed gate MIS-FETs. The device with an RTCVD SiN gate dielectric shows extremely lower current and $\mathrm{g}_{\mathrm{m}}$ compared with the one with a PE-ALD SiN gate dielectric. Fig. 10 and Fig. 11 summarize how the $I_{D}$ and $g_{m}$ peak drop when the $\mathrm{V}_{\mathrm{TH}}$ is increased. For a negative $\mathrm{V}_{\mathrm{TH}}$ of $1 \mathrm{~V}$, both devices have similar current. However, The device with an RTCVD SiN gate dielectric shows a much more pronounced current drop and $g_{m}$ degradation once the $V_{T H}$ is above $0 \mathrm{~V}$. Strongly or completely recessing the AlGaN barrier not only reduces the carrier concentration under the gate region but also brings the interface under the gate dielectric close to the channel (Fig. 12). Indeed, $\mathrm{g}_{\mathrm{m}}$ degradation could be either due to the change in carrier concentration or the change in carrier velocity [13]. However, the closer the traps to the $\mathrm{GaN}$ channel are, the stronger the electron scattering happens. So, such strong electron scattering could lead to serious mobility degradation, resulting in much more $\mathrm{g}_{\mathrm{m}}$ degradation and current drop for the device with an RTCVD SiN gate dielectric. Yet, in a device with a better quality gate dielectric, i.e., a lower $\mathrm{D}_{\mathrm{it}}$ or less border traps, the current drop can be minimized as shown in the $I_{D}-V_{D}$ characteristics comparison of a fully recessed MIS-FET with a PEALD SiN and an RTCVD SiN (Fig. 13) gate dielectric.

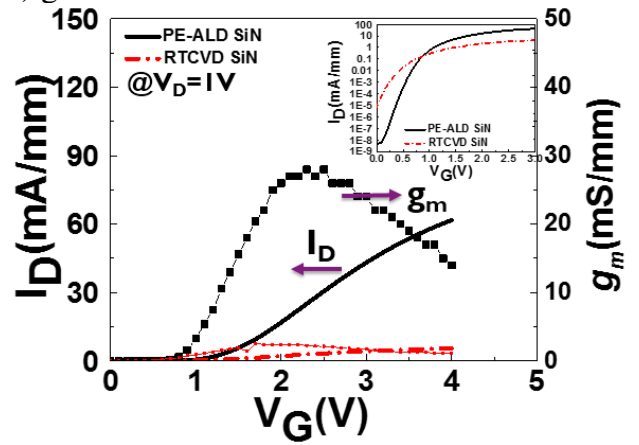

Fig. 9. $I_{D}-V_{G}$ characteristics of the GaN-MISFET devices. $I_{D}$ is below $1 \mathrm{E}-8 \mathrm{~mA} / \mathrm{mm}$ at $\mathrm{V}_{\mathrm{G}}=0 \mathrm{~V}$ (inset figure). The $\mathrm{V}_{\mathrm{TH}}$ is $1.1 \mathrm{~V}$ and $1.3 \mathrm{~V}$, respectively for a PE-ALD $\mathrm{SiN}$ and an RTCVD SiN gate dielectric.
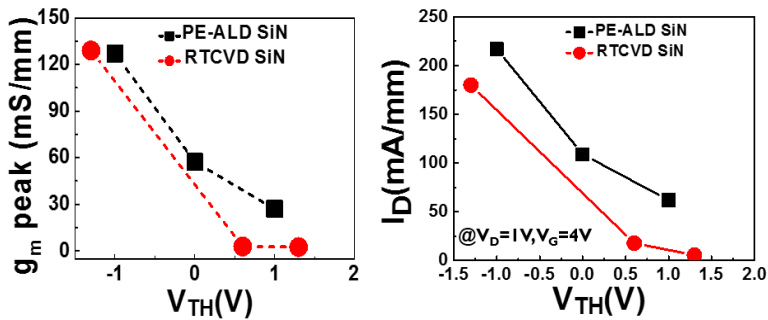

Fig. 10. $g_{m}$ peak transconductance vs. $V_{T H}$ (left) and $I_{D}$ vs. $\mathrm{V}_{\mathrm{TH}}$ (right) on devices with a PE-ALD SiN and an RTCVD $\mathrm{SiN}$ gate dielectric.

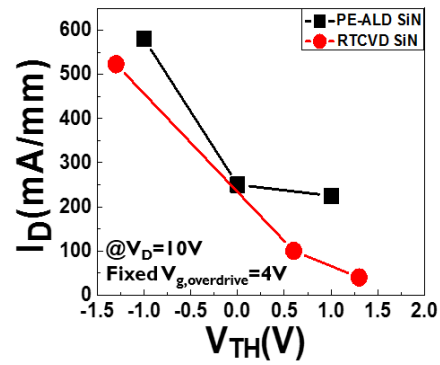

Fig. 11. $I_{D}$ for a fixed gate overdrive of $4 V$, and $V_{D}=10 V$.

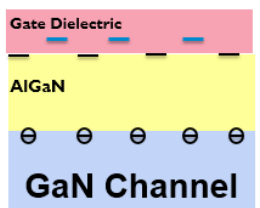

(a)

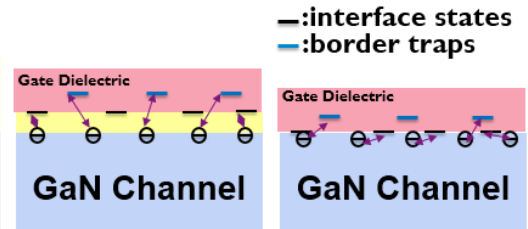

(b)
Fig. 12. A poor quality gate dielectric, such as an RTCVD $\mathrm{SiN}$, the electrons in the channel can easily be trapped by interface states or border traps. The closer these traps to the $\mathrm{GaN}$ channel are (a thinner and thinner AlGaN barrier shown from (a) to (c)), the stronger the scattering happens. This results in serious $g_{m}$ degradation and current drop (Fig. 10 and Fig. 11). However, this issue can be reduced by using a high quality gate dielectric, such as a PE-ALD SiN. 

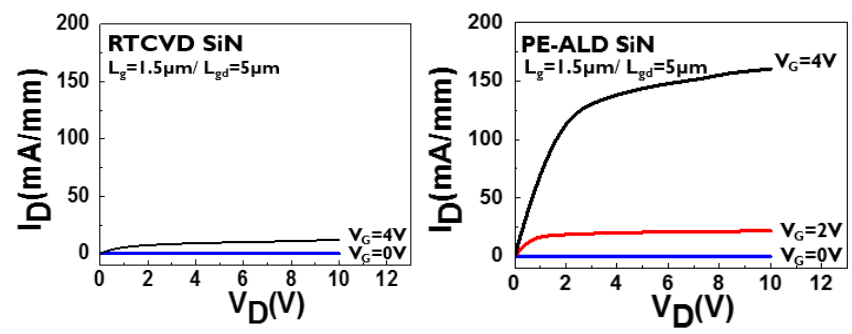

Fig. 13. $I_{D}-V_{D}$ characteristics of the E-mode MIS-FETs with an RTCVD SiN gate dielectric (a) and a PE-ALD SiN gate dielectric (b).

Furthermore, gate dielectric quality not only has a strong influence on the gate leakage current but also on time dependent dielectric breakdown (TDDB). In Fig. 14(a), the $\mathrm{I}_{\mathrm{G}^{-}}$ $\mathrm{V}_{\mathrm{G}}$ characteristics for both gate dielectrics are compared. The devices with $25 \mathrm{~nm} / 35 \mathrm{~nm}$ PE-ALD show less than $1 \mu \mathrm{A} / \mathrm{mm}$ of $\mathrm{I}_{\mathrm{G}}$ at $\mathrm{V}_{\mathrm{G}}=15 \mathrm{~V}$. Fig. 14 also shows the TDDB experiments for the devices with PE-ALD SiN. The distribution of the $t_{B D}$ statistically follows the Weibull distribution and the lifetime could be predicted with scaling to low percentage failure on devices with a large gate width (Fig. 15). For a 25nm PEALD SiN gate dielectric, an operating voltage can be determined by either an exponential law $(4.9 \mathrm{~V})$ or a power law $(7.2 \mathrm{~V})$ after scaling to $0.01 \%$ at 20 years for devices with $\mathrm{Wg}=36 \mathrm{~mm}$. This suggests the gate dielectric strength should be sufficiently high in order to have enough TDDB margin. Furthermore, TDDB margin is highly enhanced on the devices with a $35 \mathrm{~nm}$ PE-ALD gate dielectric (13V (an exponential law) or $15.5 \mathrm{~V}$ (a power law)). This could be contributed by a thick gate dielectric, which can increase the dielectric strength and reduce the possibility to form the percolation path around the recessed gate corner [14].

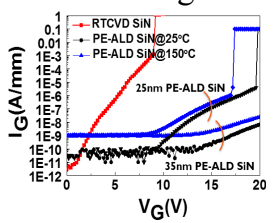

(a)

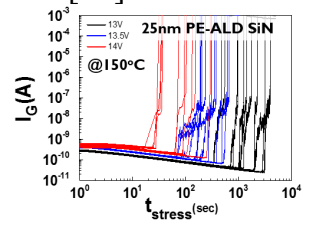

(b)

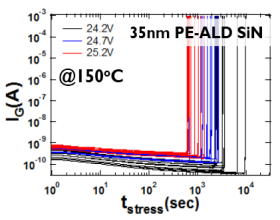

(c)
Fig. 14. (a) $I_{G}-V_{G}$ characteristics of E-mode MIS-FETs. (b) Gate current monitored with three different gate voltages (10 devices per $\left.\mathrm{V}_{\mathrm{G}}\right)$. The small gate width $(\mathrm{Wg}=10 \mu \mathrm{m})$ devices were used to study the intrinsic reliability. The calculated $\beta$ from the Weibull plot (not shown here) is $1.9(25 \mathrm{~nm}$ thickness) and 2.67 (35nm thickness), respectively.

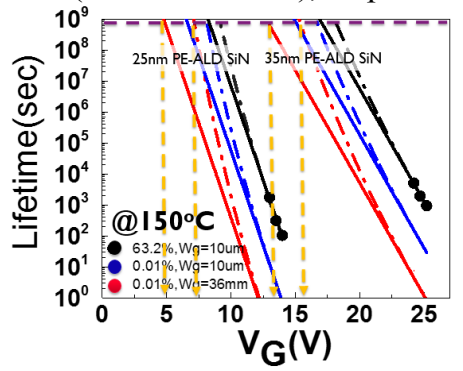

Fig. 15. Lifetime extrapolation of $\mathrm{t}_{\mathrm{BD}}$ at $150^{\circ} \mathrm{C}$ towards low bias conditions can either by an exponential law (solid line) or a power law(dash line).

\section{CONCLUSIONS}

In summary, the impact of the interface properties and the quality of the gate dielectrics have been comprehensively investigated in D-mode and E-mode recessed gate transistors. We have observed three main issues:1) the $\mathrm{V}_{\mathrm{TH}}$ hysteresis, 2) the drain current reduction for a deep gate recess, and 3) the forward gate bias TDDB reliability. The hysteresis is highly correlated with the interface states and/or border traps due to the trapping/de-trapping under a positive gate bias. When the gate dielectric interface is very close to the GaN channel, the current is strongly reduced because of the increased scattering between the electrons and the interface states/border traps, leading to $\mathrm{g}_{\mathrm{m}}$ degradation, which is probably due to mobility degradation. However, this current drop could be minimized by using a good quality gate dielectric, such as a PE-ALD SiN which has lower $\mathrm{D}_{\mathrm{it}}$ and less border traps. Finally, not only the gate leakage current but also the gate dielectric TDDB reliability is needed to be taken into account. In the case of PEALD SiN, the minimum gate dielectric thickness to provide enough gate overdrive with a sufficient TDDB margin is $25 \mathrm{~nm}$. Furthermore, a 35nm PE-ALD SiN gate dielectric can highly boost the TDDB margin.

\section{REFERENCES}

[1] M. Van Hove et al, "CMOS Process-Compatible High-Power LowLeakage AlGaN/GaN MISHEMT on Silicon," IEEE Electron Device Letters, vol. 33, no. 5, pp. 667-669, May. 2012.

[2] B. De Jaeger et $\boldsymbol{a l}$, "Au-free CMOS-compatible AlGaN/GaN HEMT processing on $200 \mathrm{~mm} \mathrm{Si} \mathrm{substrates,"} \mathrm{Proc.} \mathrm{Int.} \mathrm{Symp.} \mathrm{Power}$ Semiconductor Devices and IC's (ISPSD), pp. 49-52, 2012.

[3] W. Choi et al, "High-Voltage and Low-Leakage-Current Gate Recessed Normally-Off GaN MIS-HEMTs With Dual Gate Insulator Employing PEALD-SiNx/RF-Sputtered-HfO ," IEEE Electron Device Letters, vol. 35, no. 2, pp. 175-177, Feb. 2014.

[4] M. Wang et al, " $900 \mathrm{~V} / 1.6 \mathrm{~m} \Omega \cdot \mathrm{cm} 2$ normally off $\mathrm{Al}_{2} \mathrm{O}_{3} /$ GaN MOSFET on silicon substrate," IEEE Trans. on Electron Devices, vol. 61, no. 6, pp. 2035, Jun. 2014.

[5] T.-E. Hsieh et al, "Gate Recessed Quasi-Normally OFF Al2O3/AlGaN/GaN MIS-HEMT With Low Threshold Voltage Hysteresis Using PEALD AIN Interfacial Passivation Layer," IEEE Electron Device Letters, vol. 35, no. 7, pp.732-734, July.2014.

[6] S. Yang et al, "High-Quality Interface in $\mathrm{Al}_{2} \mathrm{O}_{3} / \mathrm{GaN} / \mathrm{AlGaN} / \mathrm{GaN}$ MIS Structures With In Situ Pre-Gate Plasma Nitridation," IEEE Electron Device Letters, vol. 34, no. 12, pp. 1497-1499, Dec. 2013.

[7] P. Moens et al, "An Industrial Process for $650 \mathrm{~V}$ rated GaN-on-Si Power Devices using in-situ SiN as a Gate Dielectric," Proc. Int. Symp. Power Semiconductor Devices and IC's (ISPSD), pp. 370-373, 2014.

[8] J.-J. Zhu et al, "Improved Interface and Transport Properties of AlGaN/GaN MIS-HEMTs With PEALD-Grown AlN Gate Dielectric," IEEE Trans. on Electron Devices, vol. 62, no. 2, pp. 512-518, Feb. 2015.

[9] W. Choi et al, "Impacts of conduction band offset and border traps on Vth instability of gate recessed normally-off GaN MIS-HEMTs," Proc. Int. Symp. Power Semiconductor Devices and IC's (ISPSD), pp. 370373, 2014.

[10] E. H. Nicollian, MOS Physics and Technology, John Wiley \& Sons, NY, 1982.

[11] X. Sun et al, "Electrical Characterization of Gate Traps in FETs With Ge and III-V Channels," IEEE Trans. Device and Material Reliability, vol. 13, pp. 463, Dec. 2013.

[12] H. B. Profijt $\boldsymbol{e t} \boldsymbol{a l}$, "Plasma-Assisted Atomic Layer Deposition: Basics, Opportunities, and Challenge," Jour. of Vac. Sci.\&Tech. A, vol. 9, pp. 05080-1-05080-26, Aug. 2011.

[13] S. Vitanov et al, "Physics-Based Modeling of GaN HEMTs," IEEE Trans. on Electron Devices, vol. 59, no. 3, pp. 685, Mar. 2012.

[14] T.-L. Wu et al, "Time Dependent Dielectric Breakdown (TDDB) Evaluation of PE-ALD SiN gate dielectrics on $\mathrm{AlGaN} / \mathrm{GaN}$ recessed gate D-mode MIS-HEMTs and E-mode MIS-FETs," IEEE International Reliability Physics Symposium (IRPS), 2015. (accepted). 